\title{
末次盛冰期以来观光木的潜在地理分布变迁
}

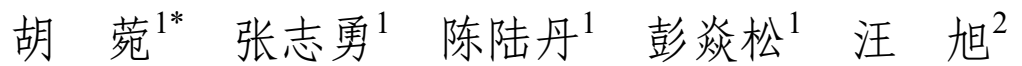 \\ ${ }^{1}$ 中国科学院庐山植物园, 江西省植物迁地保护与利用重点实验室, 江西九江 $332900 ;{ }^{2}$ 江西省林业产业发展管理局, 南昌 330006
}

\begin{abstract}
摘 要 观光木(Tsoongiodendron odorum)是木兰科的古老残遗物种, 目前正面临严峻的生存威胁，属于极小种群濒危植物。 通过生态位模型(ENM)能够重建观光木地理分布格局的历史变迁, 探究气候变化对该物种分布的影响, 并了解其地理分布与 气候需求间的关系，从而为全球变暖背景下观光木的保护提供理论基础。该文基于 96 条现代分布记录和 8 个环境变量, 采用最 大熵(MaxEnt)模型模拟观光木在末次盛冰期、全新世中期、现代和未来(2061-2080年，RCP 8.5)的潜在分布区，利用SDM toolbox分析观光木的地理空间变化, 并综合贡献率、置换重要值和Jackknife检验来评估气候因子的重要性。研究结果表明: (1) 观光木的高度适生区在南岭地区，末次盛冰期时没有大尺度向南退缩，很可能在山区避难所原地存活; (2)在全新世中期和未 来两个增温的气候情境下, 观光木的分布区均表现为缩减, 其中未来分布的减幅更大, 表明气候变暖对观光木的生长有一定 的负面影响; (3)总体上看, 观光木各个时期的地理分布范围相对稳定, 说明观光木对气候变化有一定的适应能力, 人为活动 或自身繁育问题可能是致濒的重要原因, 并建议对广东和广西群体进行优先保护。
\end{abstract}

关键词 观光木; 生态位模型; 地理分布; 末次盛冰期; 气候变化

胡苑, 张志勇, 陈陆丹, 彭炎松, 汪旭 (2020). 末次盛冰期以来观光木的潜在地理分布变迁. 植物生态学报, 44, 44-55. DOI: 10.17521/cjpe.2018.0258

\section{Changes in potential geographical distribution of Tsoongiodendron odorum since the Last Glacial Maximum}

HU Wan ${ }^{1 *}$, ZHANG Zhi-Yong ${ }^{1}$, CHEN Lu-Dan ${ }^{1}$, PENG Yan-Song ${ }^{1}$, and WANG Xu ${ }^{2}$

${ }^{1}$ Lushan Botanical Garden, Chinese Academy of Sciences, Provincial Key Laboratory of Plant ex Situ Conservation and Utilization, Jiujiang, Jiangxi 332900, China; and ${ }^{2}$ Forestry Industry Development Administration of Jiangxi Province, Nanchang 330006, China

\section{Abstract}

Aims Tsoongiodendron odorum is an ancient relic species belonging to the family Magnoliaceae, but it is labelled endangered plant with extremely small populations and facing serious threats to its wild survival now. Using Ecological Niche Modelling (ENM) to hindcast historical changes in its distribution during the Last Glacial Maximum (LGM), this study aims to explore the impact of climate change on the distribution of T. odorum, and to evaluate the relationship between species distribution and environmental variables. The results of this study could contribute to the conservation of $T$. odorum in the context of global warming.

Methods Based on 96 modern geographical distribution records and 8 bioclimatic variables, we simulated the potential distribution of T. odorum during the LGM, Mid-Holocene, present and future (period of 2061-2080 in the Representative Concentration Pathway 8.5 climate scenario) with MaxEnt model. The changes in species distribution through time were analyzed by SDM toolbox, while the importance of bioclimatic variables was evaluated by percent contribution, permutation importance and Jackknife test.

Important findings (1) The highly suitable region for T. odorum was Nanling region, and this area might be the refuge where $T$. odorum survived in situ during the LGM because only slightly southward retreat in distribution was detected in this region during the LGM. (2) In the two warming climate scenarios (Mid-Holocene and future), the area of the suitable region was reduced, and the decrease of future distribution is greater than that during the Mid-Holocene, which suggests that warming climate might have a negative impact on the distribution of $T$. odorum. (3) Overall the stability of distribution range of T. odorum in each period indicates the climate adaptation of this species. Human activity or self-breeding problem was likely the significant cause leading to endangered

收稿日期Received: 2018-10-17 接受日期Accepted: 2019-01-17

基金项目：国家自然科学基金(41961009)、江西省科研基础条件和能力建设项目(2017ZDD01002)和中国科学院科技服务网络计划(KFJ-3W-No1)。

Supported by the National Natural Science Foundation of China (41961009), the Scientific Infrastructure and Capacity Building of Jiangxi Province (2017ZDD01002), and the Science and Technology Service Network Initiative (KFJ-3W-No1).

*E-mail: hwan603@163.com 
condition. Guangdong and Guangxi should be regarded as priority conservation areas as shown by our results.

Key words Tsoongiodendron odorum; ecological niche modelling; geographical distribution; Last Glacial Maximum; climate change

$\mathrm{Hu}$ W, Zhang ZY, Chen LD, Peng YS, Wang X (2020). Changes in potential geographical distribution of Tsoongiodendron odorum since the Last Glacial Maximum. Chinese Journal of Plant Ecology, 44, 44-55. DOI: 10.17521/cjpe.2018.0258

理解和预测物种如何应对全球气候变化一直是 生物多样性研究的核心问题。气候变化使得物种的 分布地改变, 生境破碎化加剧, 种群大小减少以及 遗传多样性降低, 进而将导致植物的迁移、濒危甚 至灭绝, 尤其是对于那些生态位狭窄和种群极小的 濒危植物(Thuiller et al., 2005; Heller \& Zavaleta, 2009; Bellard et al., 2012; Diez et al., 2012; Grimm et al., 2013; Watson et al., 2013; Qu et al., 2018)。

第四纪(从2.58 Ma开始至今)以来, 地球气候的 剧烈变化对当今物种的分布格局具有重要影响 (Hewitt, 2004)。根据狍粉数据, 在末次盛冰期(Last Glacial Maximum, LGM) 全球大降温背景下, 欧亚 大陆和北美洲的植被类型向赤道方向后退, 森林面 积不同程度地减少和破碎化, 中国东部暖温带常绿 阔叶林和混交林向南推移了 300-1 000 km, 而在距 今最近的一个暖期一全新世中期, 由于气候高温 高湿, 欧亚大陆中纬度北部地区的森林带略向北移 动, 中国的暖温带常绿阔叶林和混交林也北移 200-500 km (陈瑜和倪健, 2008; Ni et al., 2010)。显 然, 气候对物种的分布范围具有深远的影响, 是大 尺度上决定物种分布的关键因素(吴建国等, 2009)。 如今, 现代物种正面临着未来气候迅速变暖的威 胁。IPCC研究表明, 近百年来全球地表平均温度已 经增长了 $0.85{ }^{\circ} \mathrm{C}$, 预计到 2100 年将持续升温 $0.3-$ $1.7{ }^{\circ} \mathrm{C}$, 最高可能增长2.6-4.8 ${ }^{\circ} \mathrm{C}$, 与此同时降水、辐 射、潜在蒸散以及其他气候变量的季节变化也将随 气候变暖而发生改变(Stocker et al., 2014)。全球变暖 显著影响物种的分布(Chapin et al., 2000; Hamann \& Wang, 2009), 例如, 20世纪以来, 欧洲西部山脉发 生了植物物种集中向山顶迁移的现象, 迁移的平均 速度每年上升近 $3 \mathrm{~m}$ (Lenoir et al., 2008); 而近30年 来的增温已经导致中国中东部 65 种植物中 $80 \%$ 种类 的分布区发生北移(宋文静等, 2016)。气候变化使植 物保护面临着巨大的不确定性(Lavergne et al., 2004; Heller \& Zavaleta, 2009), 因此, 研究植物分布格局 对气候变化的响应, 了解生物的气候需求及其与物 种地理分布间的关系, 对于揭示物种的形成、迁移
和扩散历史, 提出合理的物种多样性保护策略具有 重要意义。

生态位模型(ENM) 是利用物种现代分布记录和 气候因子, 分析分布点和环境因子相关性, 判断物 种的生态需求, 并将运算结果投射到不同的时间或 地理空间中来预测物种的实际分布和潜在分布(朱 耿平等, 2013)。运用生态位模型对现代、过去、未 来气候下植物分布进行预测, 能够为深入理解物种 进化历程以及未来迁移轨迹提供重要的参考依据 (Warren et al., 2013)。现代分布和预测的未来分布不 重叠说明种群将可能受到全球变暖的严重威胁 (Waltari et al., 2007), 而过去模型和现代分布的重 叠区域可能暗示着拥有丰富遗传多样性的避难所地 区(Provan \& Bennett, 2008; Nogués-Bravo, 2009; Thomas et al., 2012)。近30年来, 研究者们开发了 20 多种生态位模型(Elith et al., 2006; Qiao et al., 2012), 如BIOCLIM、BIOMAPPER、CLIMEX、DOMAIN、 GARP、GAM、GLM、MaxEnt等，每种模型有不同 的理论基础、分析方式和数据需求。其中最大熵 (MaxEnt)模型是目前使用最为广泛的生态位模型 (Ahmed et al., 2015; Barbosa \& Schneck, 2015; Vaz et al., 2015), MaxEnt模型根据已知的物种分布信息预 测物种的潜在适宜分布区域(Phillips et al., 2006), 模型精度要优于同类预测模型(王运生等, 2007; 邢 丁亮和郝占庆, 2011), 而且在样本量很小 $(\leqslant 20)$ 甚 至物种分布数据残缺的情况下, 该模型仍具有良好 的预测效果和可信度(Kumar \& Stohlgren, 2009), 是 针对可用分布数据较少的濒危物种适生区预测最有 效的模型(张路, 2015)。

观光木 (Tsoongiodendron odorum) 又名宿轴木 兰、香花木, 隶属木兰科的单种属观光木属, 起源古 老, 是中生代白严纪的子遗植物。尽管观光木广泛 分布在我国热带到中亚热带南部地区, 但是野生种 群非常小, 而且野生种群的数量及种群大小仍在不 断缩减(肖荣高等, 2017), 目前已被世界自然保护联 盟(IUCN)列为近危种, 被我国先后列为国家II级重 点保护植物(傅立国和金鉴明, 1992)和全国极小种 
群野生植物(国家林业局野生动植物保护与自然保 护区管理司, 2013)。观光木的研究工作主要集中在 生物量及养分循环、生理生态与系统发育、群落与 种群生态等基础生物学方面(Yang et al., 2004; 邓贤 兰等, 2012; Zhu et al., 2013; 肖荣高等, 2017)。关于 观光木的濒危机制, 近年来也开展了一些生殖生物 学及种群遗传多样性等方面的研究(王霞等, 2012; 吴雪琴等, 2013; 徐刚标等, 2014), 然而从气候的角 度探讨观光木分布和生长所受环境限制的研究十分 贫乏。Qu等(2018)利用生态位模型对观光木等6种极 小种群植物在现代及未来气候情境下的分布区进行 了预测, 结合我国现有的自然保护区分析了观光木 等植物的重点保护区域, 并提出极小种群植物的就 地/迁地保护措施需与气候变化监测相联合, 但文 中未见对观光木分布与环境气候关系的深入探析。 观光木在我国间断零星分布的现状, 与历史气候有 何关联? 气候变迁对观光木适生区分布有何影响, 主要限制因子及其耐受范围如何? 在观光木原生生 境日益遭受破坏的情况下, 从环境气候方面探明观 光木的潜在适生生境, 研究其在自然条件下对各种 环境因子的耐受能力, 可以为观光木的保护及利用 提供理论基础。因此, 本文将基于MaxEnt模型模拟 和预测观光木在末次盛冰期、全新世中期、现代
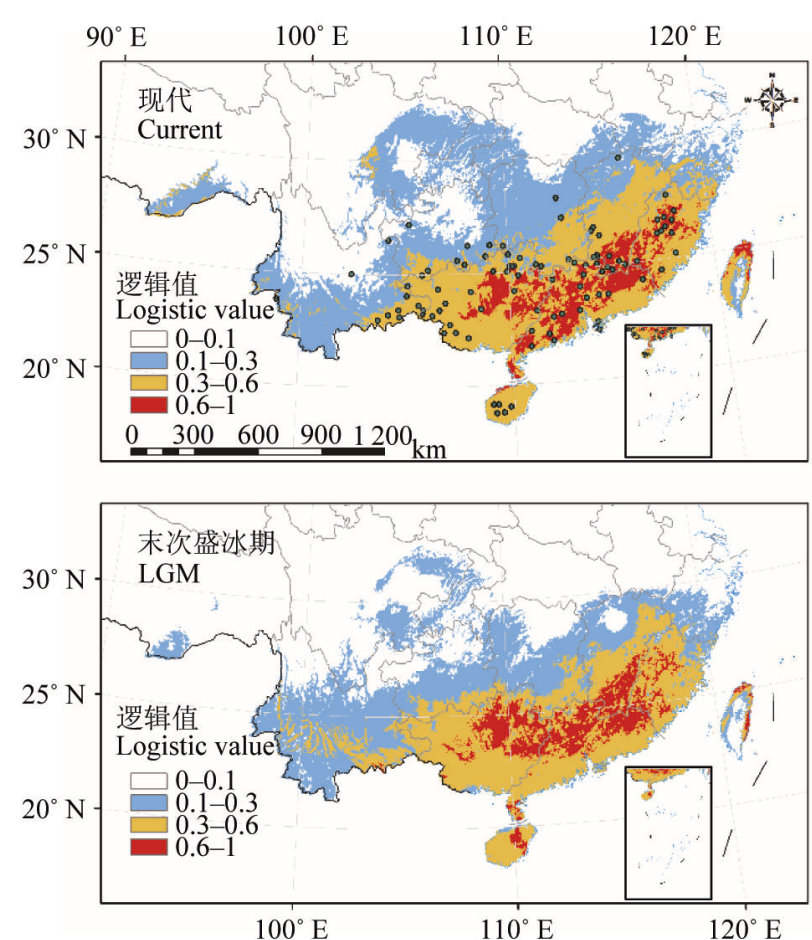

(1970-2000年)和未来(2061-2080年, 2070s) 4个历史 时期的潜在分布区，预测末次冰期气候变迁和未来 全球变暖情景下观光木的地理分布变化趋势, 探究 限制观光木分布的主要气候因子, 从而为观光木的 保护政策制定、保护区划定、引种驯化和资源管理 等方面提供科学依据。

\section{1 材料和方法}

\section{1 数据收集与处理}

通过查阅 《中国植物志》和各地方植物志以及 相关文献资料, 确定观光木种群分布点, 通过检索 全球生物多样性信息网(http://www.gbif.org)、中国 数字植物标本馆(http://www.cvh.ac.cn)、教学标本资 源共享平台(http://mnh.scu.edu.cn)、国家标本资源共 享平台 (http://www.nsii.org.cn) 和中国植物图像库 (http://www.plantphoto.cn), 分别获得观光木标本记 录67、323、80、233和636条，合计 1339 条。利用 Google Earth确定标本地理坐标，位置信息精确到 县级行政区划。去除重复记录、模糊记录和人工引 种栽培记录, 同时考虑气候数据精度为 $2.5^{\prime}$, 为降低 采样偏差引起的模型过度拟合, 在每个 $2.5^{\prime} \times 2.5^{\prime}$ 网 格中只取唯一分布点, 最终筛选得到有效分布记录 96个(图1)。
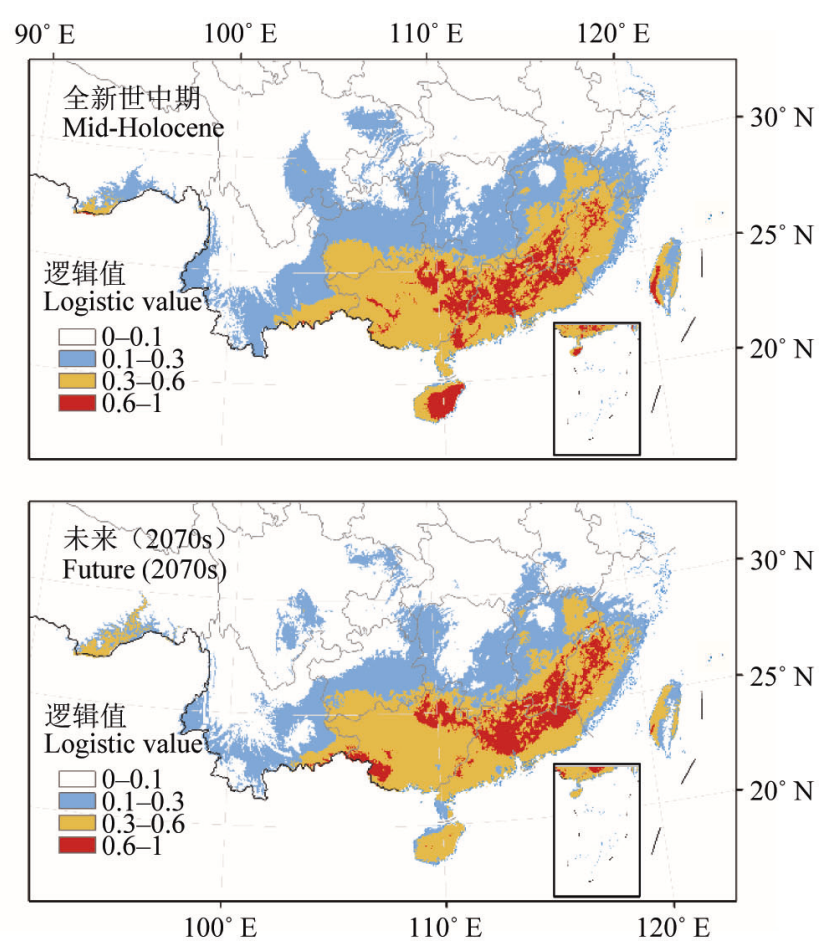

图1 观光木的现代分布点(黑色点)和MaxEnt模型预测的不同时期观光木潜在分布区。

Fig. 1 Occurrence points (black dots) and potential distribution of Tsoongiodendron odorum simulated by MaxEnt in different periods.

www.plant-ecology.com 
末次盛冰期、全新世中期、现代和未来的气候 数据均自WorldClim数据库 (http://worldclim.org)下 载, 每个时期的气候数据包括了 19 个生物气候变量 (bio01-bio19), 空间分辨率为2.5 arc-minutes。末次 盛冰期、全新世中期、未来气候数据采用通用气候 系统模式CCSM4生成, 其中未来选择最高温室气 体排放情景“典型浓度目标” 8.5 (RCP 8.5)。以国家 基础地理信息系统(http://nfgis.nsdi.gov.cn)中下载的 1:4000 000的中国行政区划矢量地图作为分析底图, 应用ArcGIS 10.2对 4 个时期的所有气候变量数据进 行剪裁, 获得各时期的中国气候图层。

\section{2 模型建立与评估}

\subsection{1 环境变量篮选}

由于预测物种分布通用的 19 个气候变量间存在 不可避免地自相关及多重线性重复等问题，这些高 度相关的变量在模型预测过程中会引入午余信息, 影响预测结果(陈新美等, 2012; 朱耿平等, 2014)。因 此在建立模型前, 需要对环境变量进行相关性分析 和節选, 提高模型的模拟精度。参考Worthington等 (2016)的方法, 首先使用现代的全部环境变量构建 初始模型(具体方法参照1.2.2), 选择MaxEnt 3.3.3软 件中的刀切法(Jackknife test)来测定环境变量对模 型预测的贡献大小, 同时利用ArcGIS 10.2软件提取 96 个分布点上 19 个气候变量的数值, 通过SPSS 13.0 软件采用Pearson相关系数 $(r)$ 检验气候变量间的多 重共线性(Yang et al., 2013), 保留相关性较小 $|r|<$ 0.8 的气候变量, 在相关系数 $|r| \geqslant 0.8$ 的变量中选
择在初始模型检验贡献率较大的一个, 最终笁选得 到 8 个气候变量参与预测(表1)。

\subsection{2 模型构建}

将观光木的 96 个分布点数据和各时期的 8 个气 候变量数据导入MaxEnt 3.3.3, Bootstrap重复运算10 次，开启Random seed，随机选取 $75 \%$ 的观光木分布 点作为训练集用于模型构建, 其余 $25 \%$ 的数据作为 测试集用于模型检验。使用绘制环境变量响应曲线 功能以评估各气候变量对模型预测结果的影响, 使 用刀切法功能判断环境变量的重要性, 测定影响物 种分布的主导因子, 其余参数保持默认设置。模型 最后以logistic output的形式输出结果, 选择10次运 算平均值的ASC文件, 栅格数值以逻辑值形式给出 物种的分布概率, 反映目标区域中的各栅格满足物 种现实生态位的程度(Phillips \& Dudík, 2008)。将其 导入ArcGIS 10.2软件, 利用重分类工具中的人工分 级法将适宜度分为 4 个等级：0-0.1 为不适生区, 0.1-0.3为低度适生区, 0.3-0.6为中度适生区, 大于 0.6 为高度适生区。

\subsection{3 模型结果评估}

MaxEnt模型软件会自动绘制受试者工作特征 曲线( $\mathrm{ROC}$ 曲线)对模拟结果进行评价, 曲线下面积 $A U C$ 值(取值范围0-1)反映预测精度, $A U C$ 值越大表 示环境变量与预测的物种地理分布模型之间的相关 性越大，模型预测结果越准确(Hanley \& McNeil, 1982 )。一般认为 $A U C$ 值为 $0.5-0.6$ 时为失败, $0.6-0.7$ 时为较差, $0.7-0.8$ 时为一般, $0.8-0.9$ 时为良好, 大于

表1 观光木分布预测使用的气候变量及其重要性参数

Table 1 Environmental variables and their importance parameters used in distribution predicting of Tsoongiodendron odorum

\begin{tabular}{lllllllllll}
\hline $\begin{array}{l}\text { 气候变量 } \\
\text { Variable }\end{array}$ & 描述 Description & $P C(\%)$ & $P I(\%)$ & $R T G_{W}$ & $R T G_{O}$ & $T G_{W}$ & $T G_{O}$ & $A U C_{W}$ & $A U C_{O}$ \\
\hline bio01 & 年平均气温 Annual mean temperature & 7.3 & 17.2 & 2.0513 & 1.7887 & 2.1173 & 1.9008 & 0.9563 & 0.9457 & \\
bio02 & 昼夜温差月均值 Mean diurnal range & 0.3 & 0.3 & 2.0753 & 1.3582 & 2.1292 & 1.4452 & 0.9566 & 0.8999 \\
bio03 & 等温性 Isothermality & 7.7 & 9.7 & 2.0030 & 0.1760 & 2.0983 & 0.1733 & 0.9550 & 0.6606 \\
bio07 & 年平均气温变化范围 Temperature annual range & 2.8 & 25.7 & 2.0548 & 1.4095 & 2.1051 & 1.5037 & 0.9554 & 0.9049 \\
bio10 & 最暖季平均气温 Mean temperature of warmest quarter & 1.2 & 2.7 & 2.0721 & 1.0464 & 2.1313 & 1.1285 & 0.9568 & 0.8768 \\
bio12 & 年降水量 Annual precipitation & 55.2 & 40.2 & 2.0415 & 1.7442 & 2.1037 & 1.8524 & 0.9555 & 0.9422 \\
bio17 & 最干季降水量 Precipitation of driest quarter & 25 & 2.4 & 2.0654 & 1.4520 & 2.1093 & 1.5128 & 0.9558 & 0.9112 \\
bio18 & 最暖季降水量 Precipitation of warmest quarter & 0.7 & 1.9 & 2.0613 & 1.4635 & 2.1283 & 1.5902 & 0.9570 & 0.9229
\end{tabular}

$P C$, 贡献率; $P I$, 置换重要值; $R T G_{W}$, 使用除该变量外所有变量的正则化训练增益; $R T G_{O}$, 单独使用该变量的正则化训练增益; $T G_{W}$, 使用除该变量外 所有变量的测试增益; $T G_{O}$, 单独使用该变量的测试增益; $A U C_{W}$, 使用除该变量外所有变量的受试者工作特征曲线下面积; $A U C_{O}$, 单独使用该变量的 受试者工作特征曲线下面积。

$P C$, percent contribution; $P I$, permutation importance; $R T G_{W}$, regularized training gain without the variable; $R T G_{O}$, regularized training gain with only the variable; $T G_{W}$, test gain without the variable; $T G_{O}$, test gain with only the variable; $A U C_{W}, A U C$ without the variable; $A U C_{O}$, $A U C$ with only the variable. 
0.9 时为优秀(Swets, 1988)。

\section{3 地理空间分析}

通过ArcGIS 10.2分别统计各个时期不同适生 区的面积, 并利用SDM toolbox 2.2工具计算观光木 不同时期的分布面积和分布中心变化 (Brown, 2014)。在ArcGIS软件中利用“Reclass”功能将观光木 4 个时期预测分布的适生区和不适生区对应的栅格 值分别修改为 1 和 0 , 然后添加SDM toolbox工具箱, 选择“SDM Tools”模块中“Universal Tools”子目录, “Distribution Changes Between Binary SDMs"工具用 于依次计算各个时期之间(LGM-Mid-Holocene、

Mid-Holocene-Current、Current-Future)的分布面积 变化, 得到分布扩张区域、稳定区域以及收缩区域, “Centroid Changes (Lines)”工具用于计算不同时期 预测分布的几何中心位移情况, 检测观光木分布区 的总体变迁趋势, 同时得到几何中心变化的矢量重 叠密度。

物种丰富度和微特有分布(单个或少数网格单 元尺度下的物种地理分布限制)可以帮助确定优先 保护区，利用 “SDM Tools”模块中 “Biodiversity Measurements”子目录的“Estimate Richness and Endemicity (WE and CWE)"工具, 以 4 个时期预测的分 布图层为输入数据叠加计算, 分辨率为 $100 \mathrm{~km}$, ArcGIS将输出物种丰富度(SR)和加权特有分布(WE) 的叠加图层, 描绘出物种丰富度和微特有分布程度 均较高的区域。

\section{4 环境变量综合分析}

运用ArcGIS 软件中的空间分析模块提取并统 计分析各适生区的气候变量参数, 结合单变量响应 曲线(模型只使用一个变量运行时, 适宜度随变量 改变的变化曲线)和边缘响应曲线(模型使用全部变 量运行, 但仅某个变量改变, 其余变量均取分布点 上的平均值时, 适宜度随变量改变的变化曲线), 确 定不同气候变量对于观光木生长的适宜范围。

综合贡献率、置换重要值和Jackknife检验来评 估各气候变量在限制观光木现代地理分布中的重要 性。3种方法的原理不同, 贡献率依赖于求取最优解 的特定算法, 把逐步修正单一要素的系数所产生的 增益值增额分配给决定该要素的环境变量, 并将其 转化为贡献率百分比; 置换重要值与具体的算法无 关, 是通过随机置换训练点集上的环境变量数值,
测定其所引起的训练 $A U C$ 值降低幅度, 经归一化为 百分比形式; Jackknife检验通过依次使用某个变量 或依次排除某个变量创建一系列模型，比较模型间 $A U C$ 值、测试增益和正则化训练增益的差异来评估 环境变量的重要性。

\section{5 多元环境相似度面和最不相似变量分析}

裁剪观光木现代适宜区的环境变量作为参考图 层, 采用多元环境相似度面(MESS)和最不相似变 量 $(\mathrm{MoD})$ 分析探讨过去和未来情境中的气候异常区 域及引起潜在地理分布改变的关键因素。多元相似 度能够反映特定时期某点的气候状况与参考图层气 候状况的相似程度, 负值表明该点至少有一个环境 变量的数值超出了参考图层对应值的范围, 称之为 气候异常点, 最大值 100 代表该点的气候完全正常。 某点的最不相似变量指的是相似度最低(即异常程 度最高)的变量(Elith et al., 2010), 异常程度最高的 环境变量很可能是引起地理分布变迁的关键因素。 该分析通过命令窗口运行 MaxEnt.jar文件中的 density.tools.Novel工具实现, 结果以ASC格式输出。

\section{2 结果和分析}

\section{1 模型的准确性评价}

基于 96 条现代分布记录和 8 个气候变量在 MaxEnt模型中对观光木现代的潜在地理分布进行 模拟, 10 次重复的平均测试 $A U C$ 值和训练 $A U C$ 值分 别为 0.965 和 0.957 ; 末次盛冰期、全新世中期以及未 来(2070s)的模拟中, $A U C$ 值也均高达 0.95 以上, 说 明预测结果极准确。

\section{2 现代潜在地理分布}

观光木现代的潜在地理分布预测逻辑值范围在 0-0.834之间，其中96条现代分布记录平均逻辑值为 0.515 , 最高值为 0.725 (福建南平), 最低值为 0.093 (云南昆明), 分别属于高度适生区、中度适生区、低 度适宜区和不适生区的分布点比例为 $37.5 \%$ 、 $50.0 \% 、 11.5 \%$ 和 $1.0 \%$ 。适生区总面积约 132.38 万 $\mathrm{km}^{2}$, 主要包括海南、广西、广东、福建、江西、湖南、 贵州、重庆、香港、澳门、台湾大部，云南、浙江、 安徽、西藏南部, 湖北东西部及四川东部。高度适 生区面积约 16.93 万 $\mathrm{km}^{2}$, 位于广西、广东、福建局 部, 江西南部, 及海南、台湾北部少数地区, 大约在 $19.48^{\circ}-27.82^{\circ} \mathrm{N}, 107.47^{\circ}-121.90^{\circ} \mathrm{E}$ 之间，分布中心 
点位于湖南城步县。

\section{3 过去和未来潜在地理分布}

在末次盛冰期, 观光木的适生区总面积相比现 代减少了 $11.02 \%$, 其中高度适生区面积减少了 $4.57 \%$ 。分布中心位于广西临桂县, 大约比现代分布 中心纬度降低了 $0.65^{\circ}$ 。在全新世中期, 观光木适生 区总面积相比现代减少了 $10.85 \%$, 其中高度适生区 面积减少了 $15.40 \%$ 。从未次盛冰期到全新世中期, 分布区中心点向东偏北 $32.28^{\circ}$ 方向位移了 $34.15 \mathrm{~km}$, 总体上西部(主要在云南)以及北部、东部边缘少量 地区分布收缩，同时北部地区边缘和台湾省中部有 零散扩张, 适生区总面积变化不大, 但高度适生区
面积减少了 1.84 万 $\mathrm{km}^{2}$ (图2A、2E)。

在未来(2070s), 预测潜在适宜分布区显著减小, 与现代相比适生区总面积减少了 $20.07 \%$, 高度适生 区面积减少了 $18.84 \%$, 分布中心位于广西兴安县。 从现代到 $2070 \mathrm{~s}$, 分布区中心点向东偏南 $71.16^{\circ}$ 方向 位移了 $62.68 \mathrm{~km}$, 纬度下降约 $0.60^{\circ}$, 分布区北部和 云南部分地区收缩明显, 仅在极少数地区轻微扩张 (图2C、2E)。

\section{4 主要环境因子分析}

从现代各级适生区的气候变量参数结果看来, 随着观光木适生等级的提高, 除最干季降水量, 其 他7个气候因子的变化范围和标准差逐级缩小(表2),
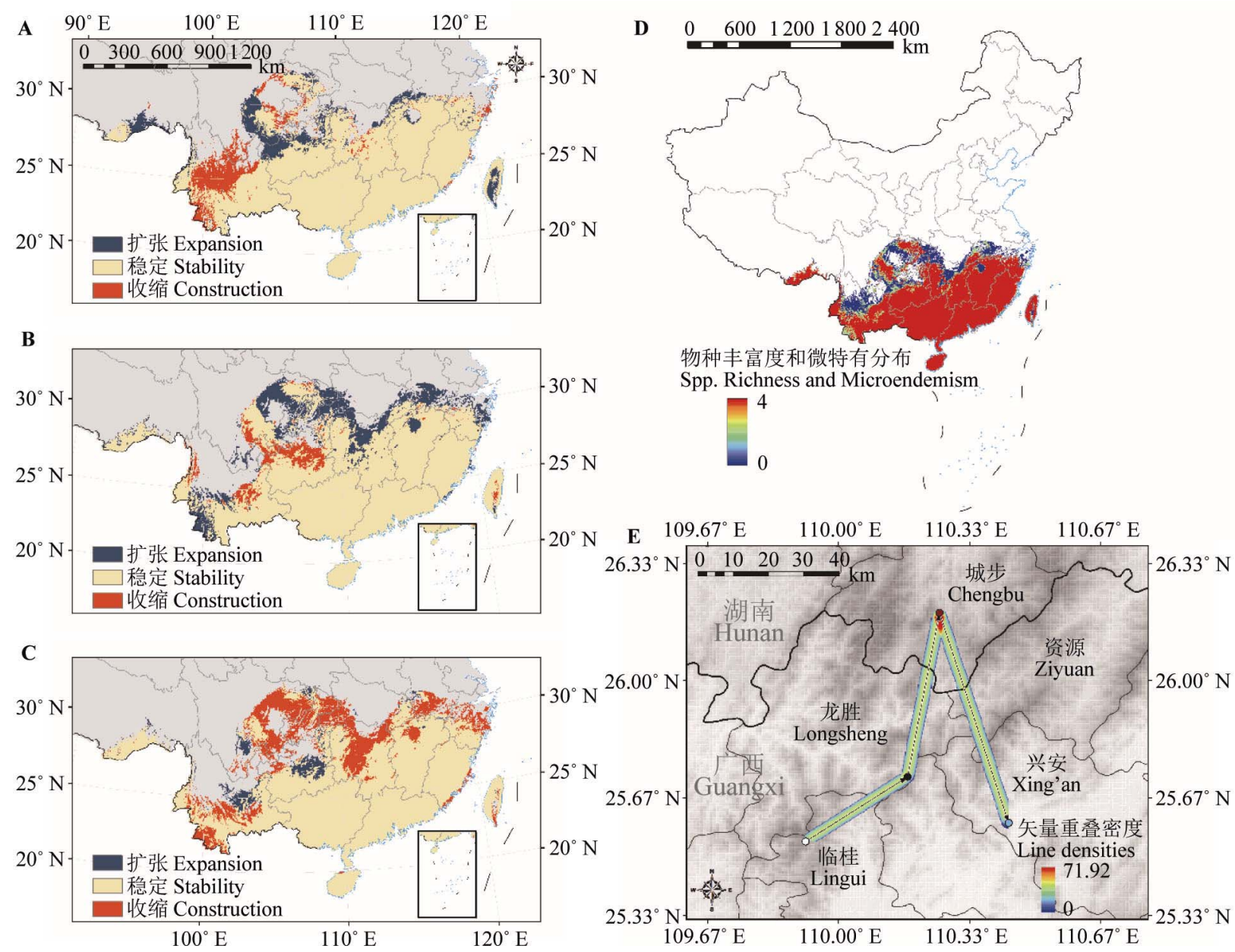

图2 不同历史时期观光木的地理分布模式。 $\mathbf{A}$, 末次盛冰期到全新世中期的分布变化。 $\mathbf{B}$, 全新世中期到现代的分布变化。 $\mathbf{C}$, 现代到未来 $2070 \mathrm{~s}$ 的分布变化。D, 四个时期物种丰富度和加权特有分布的叠加分布图。 $\mathbf{E}$, 几何中心位移以及矢量重叠密 度情况，白色圆点表示末次盛冰期几何分布中心，黑色圆点表示全新世中期几何分布中心，红色圆点表示现代几何分布中心， 蓝色圆点表示未来几何分布中心, 黑色箭头表示几何分布中心变化。

Fig. 2 Major distribution changes of Tsoongiodendron odorum between different periods simulated by MaxEnt. A, Distribution changes from the Last Glacial Maximum to Mid-Holocene. B, Distribution changes from the Mid-Holocene to Current. C, Distribution changes from the current to the future. D, Summed distributions and micro-endemism during the four periods (Last Glacial Maximum, Mid-Holocene, current and future). E, Core range shifts and the line densities, white dots represent the Last Glacial Maximum centroid, black dots represent Mid-Holocene centroid, red dots represent current centroid, blue dots represent future centroid, black arrow represent centroid change. 
表2 观光木现代各适生区的 8 个环境变量统计分析结果

Table 2 Statistics of the eight environmental variables in each of the current suitable areas for Tsoongiodendron odorum

\begin{tabular}{|c|c|c|c|c|c|c|c|c|c|c|c|c|c|}
\hline \multirow{2}{*}{$\begin{array}{l}\text { 气候 } \\
\text { 变 } \\
\text { Variable }\end{array}$} & \multicolumn{3}{|c|}{ 不适生 Unsuitable } & \multicolumn{3}{|c|}{ 低度适生 Marginally suitable } & \multicolumn{3}{|c|}{ 中度适生 Moderately suitable } & \multicolumn{3}{|c|}{ 高度适生 Highly suitable } & \multirow{2}{*}{$\begin{array}{l}\text { 单位 } \\
\text { Unit }\end{array}$} \\
\hline & $\begin{array}{c}\text { 范围 } \\
\text { Range }\end{array}$ & $\begin{array}{c}\text { 平均值 } \\
\text { Mean }\end{array}$ & $\begin{array}{l}\text { 标准偏 } \\
\text { 差 } S D\end{array}$ & $\begin{array}{c}\text { 范围 } \\
\text { Range }\end{array}$ & $\begin{array}{c}\text { 平均值 } \\
\text { Mean }\end{array}$ & $\begin{array}{l}\text { 标准偏 } \\
\text { 差 } S D\end{array}$ & $\begin{array}{l}\text { 范围 } \\
\text { Range }\end{array}$ & $\begin{array}{c}\text { 平均值 } \\
\text { Mean }\end{array}$ & $\begin{array}{l}\text { 标准偏 } \\
\text { 差 } S D\end{array}$ & $\begin{array}{c}\text { 范围 } \\
\text { Range }\end{array}$ & $\begin{array}{c}\text { 平均值 } \\
\text { Mean }\end{array}$ & $\begin{array}{l}\text { 标准偏 } \\
\text { 差 } S D\end{array}$ & \\
\hline bio01 & $-4.32-1.97$ & 4.01 & 7.14 & $4.51-4.58$ & 16.32 & 2.02 & $11.13-25.73$ & 19.14 & 2.40 & $16.26-24.65$ & 20.46 & 1.50 & ${ }^{\circ} \mathrm{C}$ \\
\hline bio02 & $2.82-0.03$ & 12.68 & 2.27 & $4.77-3.82$ & 8.07 & 0.99 & $4.07-11.91$ & 7.90 & 0.78 & $4.10-9.83$ & 8.22 & 0.63 & ${ }^{\circ} \mathrm{C}$ \\
\hline bio03 & $10.70-0.88$ & 30.45 & 5.67 & $20.57-52.24$ & 30.75 & 7.60 & $21.91-48.50$ & 31.91 & 4.36 & $25.93-39.94$ & 32.81 & 2.31 & $\%$ \\
\hline bio07 & $14.27-5.81$ & 42.49 & 8.77 & $12.24-34.58$ & 27.10 & 4.35 & $12.50-33.57$ & 25.16 & 4.11 & $11.07-31.08$ & 25.14 & 2.51 & ${ }^{\circ} \mathrm{C}$ \\
\hline bio10 & $-2.36-1.62$ & 16.87 & 7.85 & $10.26-28.62$ & 24.38 & 2.67 & $15.56-29.28$ & 26.32 & 1.90 & $20.89-29.28$ & 27.52 & 0.97 & ${ }^{\circ} \mathrm{C}$ \\
\hline bio12 & $11.00-434.00$ & 398.05 & 310.96 & $735.00-4242.19$ & 1350.65 & 297.42 & $1017.00-3501.00$ & 1589.85 & 238.43 & $1255.00-3440.00$ & 1680.38 & 241.75 & $\mathrm{~mm}$ \\
\hline bio17 & $0.00-15.00$ & 16.00 & 23.36 & $14.00-352.00$ & 95.89 & 53.68 & $26.00-723.00$ & 121.32 & 44.74 & $41.00-764.00$ & 137.18 & 56.30 & $\mathrm{~mm}$ \\
\hline bio18 & $7.00-379.00$ & 227.01 & 157.58 & $327.00-2661.00$ & 593.69 & 178.19 & $341.00-1778.00$ & 660.68 & 174.06 & $410.00-1191.00$ & 650.07 & 126.15 & $\mathrm{~mm}$ \\
\hline
\end{tabular}

表明更高适生等级对气候参数的要求也更严苛，同 时也说明最干季降水量变化对观光木的影响不明 显。根据单变量响应曲线和边缘响应曲线, 各变量 对观光木生长的最佳范围(逻辑值 0.6 以上)与高度适 生区的气候参数基本相似，单变量响应曲线基本呈 单峰型, 随着变量的增大, 适宜度不断升高, 达到 最高值后又不断降低。

环境因子重要性分析表明, 贡献率排前三位的 变量为年降水量、最干季降水量和等温性, 累积值 为 $87.9 \%$; 置换重要值排前三位的变量为年降水量、 年平均气温变化范围和年平均气温, 累积值为 83.1\% (表1)。此外，根据Jackknife检验，仅使用单独 变量时, 正则化训练增益、测试增益和 $A U C$ 值最高 的 3 个变量均依次为年平均气温、年降水量和最暖季 降水量，表明这些变量对于模型拟合具有更多的有 效信息; 使用除单独变量以外的其他变量时, 正则 化训练增益降低最多的 3 个变量依次为等温性、年降 水量和年平均气温, 测试增益降低最多的 3 个变量 依次为等温性、年降水量和年平均气温变化范围, $A U C$ 值降低最多的 3 个变量依次为等温性、年平均气 温变化范围和年降水量, 表明这些变量具有更多其
他变量没有的有效信息。综合 8 类结果排名第一的变 量来看, 影响观光木现代地理分布的气候因子主要 是年降水量(降水因子)、年平均气温和等温性(气温 因子)。

\section{5 多元环境相似度面和最不相似变量}

末次盛冰期、全新世中期和未来在现代适生区 内的多元相似度均为负值, 表明过去和未来的气候 均十分异常, 与现代气候的相似程度极低。其中, 末 次盛冰期和未来的最不相似变量均为昼夜温差月均 值和最暖季平均气温, 全新世中期的最不相似变量 为昼夜气温差月均值、最暖季平均气温和年平均气 温变化范围。总体表明, 过去和未来观光木适生区 的改变与气温因子的关系密切。

\section{3 讨论和结论}

\section{1 观光木的地理分布变迁}

MaxEnt模型的受试者工作特征曲线下面积 (AUC值)均在 0.9 以上，表明预测结果可信度极高, 可以用于探讨观光木的地理分布对第四纪气候变化 的响应。结果显示, 观光木的末次盛冰期、全新世 中期和未来的适生区面积均较现代减少(表3)。末次

表3 观光木不同时期的潜在分布区特征

Table 3 Characteristics of potential distribution in different periods for Tsoongiodendron odorum

\begin{tabular}{|c|c|c|c|c|c|c|}
\hline \multirow[t]{2}{*}{$\begin{array}{l}\text { 时期 } \\
\text { Period }\end{array}$} & \multirow{2}{*}{$\begin{array}{c}\text { 年平均气温 } \\
\text { Annual mean } \\
\text { temperature } \\
\left({ }^{\circ} \mathrm{C}\right)\end{array}$} & \multirow{2}{*}{$\begin{array}{c}\text { 年降水量 } \\
\text { Annual } \\
\text { precipitation } \\
\text { (mm) }\end{array}$} & \multicolumn{4}{|c|}{$\begin{array}{c}\text { 各适生区面积(现代相比面积变化的百分比\%) } \\
\text { Area of each suitable region (the percentage change in area compared with current, \%) }\end{array}$} \\
\hline & & & $\begin{array}{c}\text { 低度适生区 } \\
\text { Marginally suitable region }\end{array}$ & $\begin{array}{c}\text { 中度适生区 } \\
\text { Moderately suitable region }\end{array}$ & $\begin{array}{l}\text { 高度适生区 } \\
\text { Highly suitable region }\end{array}$ & $\begin{array}{c}\text { 总适生区 } \\
\text { Total suitable region }\end{array}$ \\
\hline LGM & 16.7 & 1589.7 & $64.01(-14.84)$ & $52.21(-7.87)$ & $16.16(-4.57)$ & $132.38(-11.02)$ \\
\hline Mid & 20.1 & 1741.3 & $66.93(-10.96)$ & $51.38(-9.33)$ & $14.32(-15.40)$ & $132.64(-10.85)$ \\
\hline Future & 23.1 & 1831.7 & $54.73(-27.19)$ & $50.43(-11.01)$ & $13.74(-18.84)$ & $118.91(-20.07)$ \\
\hline Current & 20.5 & 1680.4 & $75.17(0.00)$ & $56.67(0.00)$ & $16.93(0.00)$ & $148.77(0.00)$ \\
\hline
\end{tabular}

年平均气温、年降水量为各时期高度适生区的平均值。LGM, 未次盛冰期; Mid, 全新世中期; Future, 未来; Current, 现代。

Annual mean temperature and precipitation were the average values of the highly suitable region in each period. LGM, Last Glacial Maximum; Mid, Mid-Holocene.

www.plant-ecology.com 
盛冰期的分布区总面积相比现代略有减小，但高度 适生区的面积减幅不大, 分布中心在纬度上比现代 低 $0.65^{\circ}$, 总体分布范围变化不大, 说明观光木在末 次盛冰期的分布略有收缩和轻微的南移现象。尽管 化石孢粉数据显示第四纪冰期寒冷干燥的气候迫使 许多植物大幅度向南迁移避难, 常绿阔叶林的北界 向南退缩约 $1000 \mathrm{~km}\left(24^{\circ} \mathrm{N}\right)$, 热带森林植被在中国 大陆南部基本消失(Harrison et al., 2001; 陈瑜和倪 健, 2008; Ni et al., 2010)。然而大量谱系地理学研究 已经揭示了中国亚热带植物在多个避难所内原地避 难的模式, 许多植物在末次冰期预测的潜在分布区 也并未完全退缩到 $24^{\circ} \mathrm{N}$ 以南(Qiu et al., 2011; 叶俊 伟等, 2017)。观光木主要分布在亚热带南部至亚洲 热带的常绿阔叶林中, 从高度适生区的位置来看, 涵盖了南岭和武夷山山脉, 以及广西大瑶山和广东 九连山等山地区域(图1), 这些山区本身拥有高度的 地形异质性, 保持了极高的物种多样性和特有性, 通常是动植物重要的避难所(应俊生, 2001)。此外, 中国亚热带未受到冰川的直接作用, 尽管该地区年 平均气温下降4-6 ${ }^{\circ} \mathrm{C}$, 但山区受地形影响, 湿度变 化较小, 而且中国南方亚热带作为亚热带与热带的 交界地区, 可能由于气流运动形成丰富的山地降水, 山地地表的水热条件仍然适宜森林植被生长(吕燕, 2014)。因此, 可以推测观光木种群在末次盛冰期并 没有大尺度向南退缩避难, 很可能在复杂的山区地 形和温和的微环境所形成的避难所中原地保存了下 来, 并未受到冰期的不利影响或限制作用。

从末次盛冰期到全新世中期, 预测的适生区总 面积基本相同, 但全新世中期的高度适生区却有一 定程度的缩小, 同时分布中心点略微向东北方向移 动。全新世中期的气候偏暖偏湿, 东亚夏季季风的 增强导致降水强度加大, 喜温暖湿润的常绿阔叶林 有小幅度向北推进的现象, 但同时由于降水伴随的 低云量增多、夏季生长日射减少等原因, 西部常绿 阔叶林在这一时期也有明显退缩(中国第四纪狍粉 数据库小组, 2000)。从观光木在全新世中期的适生 区可以看出, 西部云南地区分布收缩明显, 广西部 分地区的高度适生区转变为中度适生区也是这一时 期高度适生区面积减少的主要原因(图1, 图2B), 因 此这一结果很可能与上述原因密切相关。可以推测, 观光木一般分布在300-1000 m的低海拔地带, 虽 喜温暖湿润的环境, 但对温湿度的要求较高, 如从
年降水量的单变量响应曲线来看, 曲线呈单峰型, 年降水量在 $2000 \mathrm{~mm}$ 左右的适宜度达到最高值, 增 大或降低年降水量其适宜度都不断下降。

未来由大气 $\mathrm{CO}_{2}$ 增加引起的全球增温与全新世 中期的增温效应有所类似(郑益群等, 2004), 从各个 时期适生区面积变化图可以发现, 现代相对全新世 中期的扩张区域，与未来相对现代的收缩区域基本 相同, 反之现代相对全新世中期的收缩区域, 与未 来相对现代的扩张区域也很相近，可见未来与全新 世中期的气候环境十分相似, 对观光木分布的影响 有一定程度的类似。两个增温的气候情境下观光木 的分布区均表现为缩减, 尤其是高度适生区都显著 减小, 但其中未来的减幅更大, 说明气候变暖对观 光木的生长有一定的负面影响, 并且未来将导致观 光木的生境减少远甚于历史时期。在未来情景下, 中国区域与全球变化情况一致, 将持续增温, 降水 出现区域性增加, 有气候预估认为, 中高纬地区较 低纬地区增温明显, 降水量除一些亚热带地区外大 部分区域都有所增加(张雪芹等, 2008), 根据观光木 未来的适生区预测, 分布区北缘收缩明显, 广西西 南部少数地区适宜度升高, 高度适生区仍相对集中 在南岭和武夷山一带(图1, 图2C), 同样可以说明观 光木分布对于温度和降水范围的严苛性, 高温和高 降水量并不适合观光木的生长。此外随着全球气候 变暖, 许多动植物有向高纬度和高海拔地区迁移的 趋势(Sekercioglu et al., 2008; 方精云等, 2018), 而 预测的观光木未来几何分布中心将向低纬度下移约 $0.60^{\circ}$, 虽然可能是由于北方分布区缩减造成, 但可 以看出观光木没有随气候变暖而向高纬度地区迁移 的趋向。可见观光木潜在分布的北界受气候限制明 显, 温暖气候情境将导致北方分布区生境适应性下 降，同时限制分布区进一步向北的扩张。当然气候 变暖不仅是温度升高和降水格局变化, 还涉及 $\mathrm{CO}_{2}$ 浓度升高、臭氧层变薄等过程, 限制植物分布的也 不仅是气候, 繁殖、竞争等生物因素也均需考虑(王 娟和倪健, 2006), 要兼顾植物群体生理生态变化和 生态系统整体变化等因素才能对观光木未来的潜在 分布区做出精准预测。

总体上看，从过去到未来 3 个时期不同情景的 气候模式下，观光木的预测分布区并没有大幅度的 缩减现象, 适生区总面积降幅有限, 缩减程度最大 的未来分布降幅为 $20.07 \%$ 。也就是说, 随着历史气 
候变迁, 观光木经历了寒冷恶劣的冰期和温暖湿润 的间冰期, 并可能即将面临高度温室效应的未来, 但其分布区总体还是相对稳定的。观光木是我国珍 稀濒危植物, 探究其濒危机制是保护生物学的核心 工作, 研究表明, 尽管观光木的野生种群很小, 但 其物种和种群仍具有较高水平的遗传变异, 说明遗 传多样性并不是观光木濒危的主要原因, 人为活动 或自身繁育问题可能是其濒危的重要原因(徐刚标 等, 2014; 肖荣高等, 2017)。本文的结果同样支持这 一结论, 观光木地理分布相对稳定并且广泛, 各时 期间分布区未变化的面积在125-136 $\mathrm{km}^{2}$ 之间，而 且其高度适生范围在南岭地区十分稳定, 说明其对 气候变化的适应能力较强, 并没有因剧烈的气候波 动而出现极端消退的现象, 仍具有广阔适宜的自然 生境。

\section{2 观光木保护的建议}

观光木的适生区分布很广, 这与其现实分布情 况十分一致, 并且观光木能够相对稳定地应对气候 变迁, 同时具有较高的遗传多样性, 然而其野外分 布却十分稀少和零散, 说明观光木的适宜生境其实 是广阔的, 对气候的适应性也较强, 而人为的生境 破坏, 以及物种本身的自然繁育问题是观光木保护 所要解决的重点, 这些结果可以为观光木的保护提 供科学的参考依据。

当然, 优先保护单元的确定, 对于制定气候变 迁下的物种保护策略十分重要。观光木现代的总体 适生分布中心点位于湖南城步县, 处于南亚热带地 区, 高度适生区主要集中在广东和广西, 包含南岭、 大瑶山、九连山、武夷山南部等山区, 此外, 物种丰 富度和加权特有分布的叠加图层(图2D)显示, 物种 丰富度和微特有分布程度均较高的区域主要位于广 东、广西、福建、海南、江西、湖南、四川和云南 等地, 说明观光木的现代分布重心位于亚热带南部 地区, 尤其在南岭南部的广东和广西地区适生度最 高, 建议将广东及广西作为观光木的优先保护区域, 这与 $\mathrm{Qu}$ 等(2018)利用地带保护规划软件计算的观光 木优先保护单元一致。对于广东及广西地区的群体 应尽可能地进行就地保护, 严格保护和修复原生生 境, 阻止人为破坏和干扰。而位于高度适生区以外 的观光木群体, 尤其是数量极少的小群体, 仅剩下 几株甚至1株, 且常散生在林缘, 山区村庄及房前屋 后的边缘个体, 存在生境片段化和地理隔离的风险,
建议进行迁地保护, 文中适宜的气候因子数据可作 为环境评估参考。此外, 值得注意的是各个时期几 何中心变化的矢量重叠密度, 矢量重叠的高密度区 域很可能是生物迁移至适宜生境过程中至关重要的 区域(Brown \& Yoder, 2015)。观光木4个时期分布中 心转变过程中的矢量重叠密度最高的区域在现代分 布中心点附近(图2E), 位于湖南城步县, 地处湘桂 之间的大南山主体附近, 这个矢量重叠的高密度区 域很可能是观光木在未来环境变化中有利于群体迁 移的通道口, 观光木在未来的适生面积减少最多, 因此建议这一区域应当重点关注和保护, 可建立自 然保护小区维持其自然生境。

\section{参考文献}

Ahmed SE, Mcinerny G, O’Hara K, Harper R, Salido L, Emmott S, Joppa LN (2015). Scientists and software-surveying the species distribution modelling community. Diversity and Distributions, 21, 258-267.

Barbosa FG, Schneck F (2015). Characteristics of the top-cited papers in species distribution predictive models. Ecological Modelling, 313, 77-83.

Bellard C, Bertelsmeier C, Leadley P, Thuiller W, Courchamp F (2012). Impacts of climate change on the future of biodiversity. Ecology Letters, 15, 365-377.

Brown JL (2014). SDMtoolbox: A python-based GIS toolkit for landscape genetic, biogeographic and species distribution model analyses. Methods in Ecology and Evolution, 5, 694-700.

Brown JL, Yoder AD (2015). Shifting ranges and conservation challenges for lemurs in the face of climate change. Ecology and Evolution, 5, 1131-1142.

Chapin III FS, Zavaleta ES, Eviner VT, Naylor RL, Vitousek PM, Reynolds HL, Hooper DU, Lavorel S, Sala OE, Hobbie SE, Mack MC, Díaz S (2000). Consequences of changing biodiversity. Nature, 405, 234-242.

Chen XM, Lei YC, Zhang XQ, Jia HY (2012). Effects of sample sizes on accuracy and stability of maximum entropy model in predicting species distribution. Scientia Silvae Sinicae, 48(1), 53-59. [陈新美, 雷渊才, 张雄清, 贾宏 炎 (2012). 样本量对MaxEnt模型预测物种分布精度和 稳定性的影响. 林业科学, 48(1), 53-59.]

Chen Y, Ni J (2008). Quantitative palaeovegetation reconstruction at lager scale based on the pollen records. Journal of Plant Ecology (Chinese Version), 32, 1201-1212. [陈瑜, 倪健 (2008). 利用狍粉记录定量重建大尺度古植被格 局. 植物生态学报, 32, 1201-1212.]

Deng XL, Wu Y, Lai MY, Xu B, Long WW (2012). Studies on the characteristics of Michelia odora population and its community in south-central Jiangxi. Guihaia, 32, 179-184.

www.plant-ecology.com 
[邓贤兰, 吴杨, 赖弥源, 许斌, 龙婉婉 (2012). 江西中 南部观光木种群及所在群落特征研究. 广西植物, 32, 179-184.]

Department of Wildlife Conservation and Nature Reserve Management, the National Forestry Bureau (2013). Rare and Endangered Plants in China. China Forestry Publishing House, Beijing. [国家林业局野生动植物保护与自然 保护区管理司 (2013). 中国珍稀濒危植物图鉴. 中国林 业出版社, 北京.]

Diez JM, D’Antonio CM, Dukes JS, Grosholz ED, Olden JD, Sorte CJB, Blumenthal DM, Bradley BA, Early R, Ibáñez I, Jones SJ, Lawler JJ, Miller LP (2012). Will extreme climatic events facilitate biological invasions? Frontiers in Ecology and the Environment, 10, 249-257.

Elith J, Graham CH, Anderson RP, Dudík M, Ferrier S, Guisan A, Hijmans RJ, Huettmann F, Leathwick JR, Lehmann A, Li J, Lohmann LG, Loiselle BA, Manion G, Moritz C, Nakamura M, Nakazawa Y, Overton JM, Peterson AT, Phillips SJ, Richardson K, Scachetti-Pereira R, Schapire RE, Soberón J, Williams S, Wisz MS, Zimmermann NE (2006). Novel methods improve prediction of species' distributions from occurrence data. Ecography, 29, 129-151.

Elith J, Kearney M, Phillips S (2010). The art of modelling range-shifting species. Methods in Ecology and Evolution, $1,330-342$.

Fang JY, Zhu JL, Shi Y (2018). The responses of ecosystems to global warming. Chinese Science Bulletin, 63, 136-140. [方精云, 朱江玲, 石岳 (2018). 生态系统对全球变暖 的响应. 科学通报, 63, 136-140.]

Fu LG, Jin JM (1992). Chinese Red Data Book of Rare and Endangered Plants. Science Press, Beijing. 454-455. [傅 立国，金鉴明 (1992). 中国植物红皮书——稀有濒危植 物. 科学出版社, 北京. 454-455.]

Grimm NB, Chapin III FS, Bierwagen B, Gonzalez P, Groffman PM, Luo Y, Melton F, Nadelhoffer K, Pairis A, Raymond PA, Schimel J, Williamson CE (2013). The impacts of climate change on ecosystem structure and function. Frontiers in Ecology and the Environment, 11, 474-482.

Hamann A, Wang T (2009). Potential effects of climate change on ecosystem and tree species distribution in British Columbia. Ecology, 39, 2773-2786.

Hanley JA, McNeil BJ (1982). The meaning and use of the area under a receiver operating characteristic (ROC) curve. $R a-$ diology, 143, 29-36.

Harrison SP, Yu G, Takahara H, Prentice IC (2001). Palaeovegetation (Communications arising): Diversity of temperate plants in east Asia. Nature, 413, 129-130.

Heller NE, Zavaleta ES (2009). Biodiversity management in the face of climate change: A review of 22 years of recommendations. Biological Conservation, 142, 14-32.

Hewitt GM (2004). Genetic consequences of climatic oscillations in the Quaternary. Philosophical Transactions of the Royal Society of London. Series B: Biological Sciences, 359, 183-195.

Kumar S, Stohlgren TJ (2009). Maxent modeling for predicting suitable habitat for threatened and endangered tree Canacomyrica monticola in New Caledonia. Journal of Ecology and the Natural Environment, 1, 94-98.

Lavergne S, Thompson JD, Garnier E, Debussche M (2004). The biology and ecology of narrow endemic and widespread plants, a comparative study of trait variation in 20 congeneric pairs. Oikos, 107, 505-518.

Lenoir J, Gégout JC, Marquet PA, De Ruffray P, Brisse H (2008). A significant upward shift in plant species optimum elevation during the 20th century. Science, 320, $1768-1771$.

Lü Y (2014). Discussion of Ancient Vegetation and Climate at 10-40 ka BP in the Subtropical Mountains of Southern China. Master degree dissertation, Nanjing Normal University, Nanjing. [吕燕 (2014). 中国南方亚热带山地 10-40 ka BP古植被与古气候特征探讨. 硕士学位论文, 南京师范大学, 南京.]

Members of China Quaternary Pollen Data Base (2000). Pollenbased biome reconstruction at Middle Holocene (6 ka BP) and last glacial maximum (18 ka BP) in China. Acta Botanica Sinica, 42, 1201-1209. [中国第四纪狍粉数据 库小组 (2000). 中国中全新世(6 ka BP)和末次盛冰期 (18 ka BP)生物群区的重建. 植物学报, 42, 1201-1209.]

Ni J, Yu G, Harrison SP, Prentice IC (2010). Palaeovegetation in China during the late Quaternary, biome reconstructions based on a global scheme of plant functional types. $\mathrm{Pa}$ laeogeography Palaeoclimatology Palaeoecology, 289, 44-61.

Nogués-Bravo D (2009). Predicting the past distribution of species climatic niches. Global Ecology and Biogeography, 18, 521-531.

Phillips SJ, Anderson RP, Schapire RE (2006). Maximum entropy modeling of species geographic distributions. Ecological Modelling, 190, 231-259.

Phillips SJ, Dudík M (2008). Modeling of species distributions with Maxent, new extensions and a comprehensive evaluation. Ecography, 31, 161-175.

Provan J, Bennett KD (2008). Phylogeographic insights into cryptic glacial refugia. Trends in Ecology \& Evolution, 23, 564-571.

Qiao H, Lin C, Ji L, Jiang Z (2012). mMWeb-An online platform for employing multiple ecological niche modeling algorithms. PLOS ONE, 7, e43327. DOI: 10.1371/journal.pone.0043327.

Qiu YX, Fu CX, Comes HP (2011). Plant molecular phylogeography in China and adjacent regions, tracing the genetic imprints of Quaternary climate and environmental change in the world's most diverse temperate flora. 
Molecular Phylogenetics \& Evolution, 59, 225-244.

Qu H, Wang CJ, Zhang ZX (2018). Planning priority conservation areas under climate change for six plant species with extremely small populations in China. Nature Conservation, 25, 89-106.

Sekercioglu CH, Schneider SH, Fay JP, Loarie SR (2008). Climate change, elevational range shifts, and bird extinctions. Conservation Biology, 22, 140-150.

Song WJ, Wu SH, Tao ZX, Dai JH (2016). Distribution change of plants over mid-eastern China during last 30 years. Geographical Research, 35，1420-1432. [宋文静, 吴绍 洪, 陶泽兴, 戴君虎 (2016). 近30年中国中东部地区植 物分布变化. 地理研究, 35, 1420-1432.]

Stocker TF, Qin D, Plattner GK, Tignor MMB, Allen SK, Boschung J, Nauels A, Xia Y, Bex V, Midgley PM (2014). Climate Change 2013: The Physical Science Basis. Cambridge University Press, Cambridge, UK.

Swets JA (1988). Measuring the accuracy of diagnostic systems. Science, 240, 1285-1293.

Thomas E, van Zonneveld M, Loo J, Hodgkin T, Galluzzi G, van Etten J (2012). Present spatial diversity patterns of Theobroma cacao L. in the neotropics reflect genetic differentiation in pleistocene refugia followed by humaninfluenced dispersal. PLOS ONE, 7, e47676. DOI: 10.1371/journal.pone.0047676.

Thuiller W, Lavorel S, Araújo MB, Sykes MT, Prentice IC (2005). Climate change threats to plant diversity in Europe. Proceedings of the National Academy of Sciences of the United States of America, 102, 8245-8250.

Vaz UL, Cunha HF, Nabout JC (2015). Trends and biases in global scientific literature about ecological niche models. Brazilian Journal of Biology, 75, 17-24.

Waltari E, Hijmans RJ, Peterson AT, Nyári ÁS, Perkins SL, Guralnick RP (2007). Locating pleistocene refugia: Comparing phylogeographic and ecological niche model predictions. PLOS ONE, 2, e563. DOI: 10.1371/journal. pone. 0000563 .

Wang J, Ni J (2006). Review of modelling the distribution of plant species. Journal of Plant Ecology (Chinese Version), 30, 1040-1053. [王娟, 倪健 (2006). 植物种分布的模拟 研究进展. 植物生态学报, 30, 1040-1053.]

Wang X, Wang J, Jiang JH, Kang M (2012). Genetic diversity and the mating system in a fragmented population of Tsoongiodendron odorum. Biodiversity Science, 20, 676-684. [王霞, 王静, 蒋敬虎, 康明 (2012). 观光木片 断化居群的遗传多样性和交配系统. 生物多样性, 20 , 676-684.]

Wang YS, Xie BY, Wan FH, Xiao QM, Dai LY (2007). Application of ROC curve analysis in evaluating the performance of alien species potential distribution models. Biodiversity Science, 15, 365-372. [王运生, 谢丙炎, 万方浩, 肖启明, 戴良英 (2007). ROC曲线分析在评价入侵物种
分布模型中的应用. 生物多样性, 15, 365-372.]

Warren R, Vanderwal J, Price J (2013). Quantifying the benefit of early climate change mitigation in avoiding biodiversity loss. Nature Climate Change, 3, 678-682.

Watson JEM, Iwamura T, Butt N (2013). Mapping vulnerability and conservation adaptation strategies under climate change. Nature Climate Change, 3, 989-994.

Worthington TA, Zhang T, Logue DR, Mittelstet AR, Brewer SK (2016). Landscape and flow metrics affecting the distribution of a federally-threatened fish, improving management, model fit, and model transferability. Ecological Modelling, 342, 1-18.

Wu JG, Lv JJ, Ai L (2009). The impacts of climate change on the biodiversity: Vulnerability and adaptation. Ecology and Environmental Sciences, 18,693-703. [吴建国, 吕佳 佳, 艾丽 (2009). 气候变化对生物多样性的影响, 脆弱 性和适应. 生态环境学报, 18, 693-703.]

Wu XQ, Xu GB, Liang Y, Sheng XB (2013). Genetic diversity of natural and planted populations of Tsoongiodendron odorum from the Nanling Mountains. Biodiversity Science, 21，71-79. [吴雪琴, 徐刚标, 梁艳, 申响保 (2013). 南 岭地区观光木自然和人工迁地保护种群的遗传多样性. 生物多样性, 21, 71-79.]

Xiao RG, Zhang YM, Wu LF, Shu ZF, Song XJ (2017). Research status and prospect of the endangered plant species Tsoongiodendron odorum Chun. Journal of Green Science and Technology, (21), 106-107. [肖荣高, 张应明, 吴林 芳, 束祖飞, 宋相金 (2017). 濒危植物观光木的研究现 状及展望. 绿色科技, (21), 106-107.]

Xing DL, Hao ZQ (2011). The principle of maximum entropy and its applications in ecology. Biodiversity Science, 19, 295-302. [邢丁亮, 郝占庆 (2011). 最大熵原理及其在 生态学研究中的应用. 生物多样性, 19, 295-302.]

Xu GB, Wu XQ, Jiang GX, Hu SL, Liu XS, Xiao YF, Hao BB (2014). Genetic diversity and population structure of an endangered species: Tsoongiodendron odorum Chun. Journal of Plant Genetic Resources, 15, 255-261. [徐刚 标, 吴雪琴, 蒋桂雄, 胡尚力, 刘雄盛, 肖玉菲, 郝博搏 (2014). 濒危植物观光木遗传多样性及遗传结构分析. 植物遗传资源学报, 15, 255-261.]

Yang XQ, Kushwaha SPS, Saran S, Xu J, Roy PS (2013). Maxent modeling for predicting the potential distribution of medicinal plant, Justicia adhatoda L. in Lesser Himalayan foothills. Ecological Engineering, 51, 83-87.

Yang YS, Chen GS, Guo JF, Lin P (2004). Decomposition dynamic of fine roots in a mixed forest of Cunninghamia lanceolata and Tsoongiodendron odorum in mid-subtropics. Annals of Forest Science, 61, 65-72.

Ye JW, Zhang Y, Wang XJ (2017). Phylogeographic history of broad-leaved forest plants in subtropical China. Acta Ecologica Sinica, 37, 5894-5904. [叶俊伟, 张阳, 王晓娟 (2017). 中国亚热带地区阔叶林植物的谱系地理历史.

www.plant-ecology.com 
生态学报, 37, 5894-5904.]

Ying JS (2001). Species diversity and distribution pattern of seed plants in China. Biodiversity Science, 9, 393-398. [应 俊生 (2001). 中国种子植物物种多样性及其分布格局. 生物多样性, 9, 393-398.]

Zhang L (2015). Applications of maximum entropy (MAXENT) model in prediction of species potential distribution ranges. Bulletin of Biology, 50, 9-12. [张路 (2015). MAXENT最大熵模型在预测物种潜在分布范围方面的 应用. 生物学通报, 50, 9-12.]

Zhang XQ, Peng LL, Lin CH (2008). Progress on the projections of future climate change with variable emission scenarios. Advances in Earth Science, 23, 174-185. [张雪芹, 彭莉莉, 林朝晖 (2008). 未来不同排放情景下气候变化 预估研究进展. 地球科学进展, 23, 174-185.]

Zheng YQ, Yu G, Xue B, Wang SM (2004). Simulations of east Asian climate at 6 ka B.P. Quaternary Sciences, 24, 28-38. [郑益群, 于革, 薛滨, 王苏民 (2004). 6 ka B.P. 东亚区域气候模拟及其变化机制探讨. 第四纪研究, 24 ,
28-38.]

Zhu GP, Liu GQ, Bu WJ, Gao YB (2013). Ecological niche modeling and its applications in biodiversity conservation. Biodiversity Science, 21, 90-98. [朱耿平, 刘国卿，卜文 俊, 高玉葆 (2013). 生态位模型的基本原理及其在生物 多样性保护中的应用. 生物多样性, 21, 90-98.]

Zhu GP, Liu Q, Gao YB (2014). Improving ecological niche model transferability to predict the potential distribution of invasive exotic species. Biodiversity Science, 22, 223-230. [朱耿平, 刘强, 高玉葆 (2014). 提高生态位模型转移 能力来模拟入侵物种的潜在分布. 生物多样性, 22 , 223-230.]

Zhu T, Meng T, Zhang J, Yin Y, Cai Z, Yang W, Zhong W (2013). Nitrogen mineralization, immobilization turnover, heterotrophic nitrification, and microbial groups in acid forest soils of subtropical China. Biology and Fertility of Soils, 49, 323-331.

责任编委: 康慕谊 责任编辑: 李 敏 